CASE REPORT

\title{
Intramuscular haemangioma of the thigh in a basketball player
}

\author{
R A Brown, K Crichton, G M Malouf
}

Br J Sports Med 2004;38:346-348. doi: 10.1136/bjsm.2003.004671

Haemangioma is a common benign soft tissue tumour. Intramuscular haemangiomas may present as a perceived sporting injury. Magnetic resonance imaging is the investigation of choice. Intramuscular haemangioma should be considered in the differential diagnosis of unexplained pain and swelling in a muscle.

$\mathrm{H}$ aemangiomas are common benign soft tissue tumours, comprising $7-10 \%$ of all soft tissue tumours. Cutaneous haemangiomas, the most common, ${ }^{1}$ are usually found in childhood. Intramuscular haemangiomas may present later in life as a cause of persistent symptoms and may present as a perceived sporting injury. Diagnostic ultrasound is an appropriate initial imaging modality for suspected haemangioma, although magnetic resonance imaging is the investigation of choice. Intramuscular haemangioma should be considered in the differential diagnosis of unexplained pain and swelling in a muscle.

\section{CASE REPORT}

A 14 year old boy presented with persistent right suprapatellar pain and swelling during basketball. It was most noticeable when he was performing lay ups and after direct trauma. The symptoms had been present for about two years. The pain had never forced him to stop playing but he would noticeably limp. The signs and symptoms would settle with rest. There was no history of major trauma, instability, or locking. His medical history was unremarkable apart from discolouration/bruising on his right lateral thigh. It was noted that over the preceding year he had been through a growth spurt.

On the day of presentation, his knee was minimally symptomatic. There was tenderness over the quadriceps tendon proximal to the insertion. There was no pain with resisted quadriceps contraction, no effusion, and ligament testing was stable. There were no signs of patellofemoral irritability. On examination of the right lateral thigh, superficial dilated veins were noted.

The differential diagnosis included suprapatellar bursitis, quadriceps tendinopathy, synovial dysplasia, and extraarticular soft tissue tumour.

The patient was advised to present for an $x$ ray examination and diagnostic ultrasound of the area when it was swollen. The radiographic results were unremarkable. An ultrasound revealed a $14 \mathrm{~cm} \times 8 \mathrm{~cm}$ venous haemangioma in the distal vastus medialis and rectus femoris which was thought not to communicate with the lateral thigh haemangioma, which was beneath the lateral thigh discolouration.

The patient was referred to a vascular surgeon whose opinion was that the lesion was too big to excise. The patient was then referred for compression sclerotherapy of both lesions with foamed sclerosant (sodium tetradecyl sulphate (STS)).

\section{DISCUSSION}

Haemangiomas are either a neoplasm or a hamartoma. A neoplasm is a new growth of cells. Most believe that haemangiomas are hamartomas, which are developmental anomalies, the cells being natural to the area and presenting in abnormal numbers.

Haemangiomas can be divided into seven types ${ }^{1}$ and may be localised or diffuse (more common ${ }^{2}$ ):

- Capillary- $50 \%^{3}$

- Cavernous

- Venous

- Arteriovenous

- Epithelioid

- Granulation tissue type

- Miscellaneous

In a 570 case series by Geschickter and Keasbey referred to by McNeill and Ray, ${ }^{4} 370$ were cutaneous or mucosal and 10 were intramuscular. Intramuscular haemangiomas make up $0.8 \%$ of all haemangiomas. ${ }^{5}$

The natural history of haemangiomas is to enlarge slowly. Their growth may be accelerated with a growth spurt or trauma. They can spontaneously regress. Malignant transformation is rare. ${ }^{1}$ They are usually detected early. Despite intramuscular lesions being concealed, 94\% present before the age of $30 .^{26}$ Some reports suggest that both sexes are equally affected ${ }^{2} 6$ although others indicate that the incidence is higher in women and girls. ${ }^{7}$ Intramuscular haemangiomas are more common in the lower limbs $(42-45 \%) .{ }^{26}$ The thigh is the most common intramuscular site (17-19\%). ${ }^{26}$ Wild et $a l^{3}$ found the quadriceps to be affected in five out of 11 cases of intramuscular haemangioma. Some $72 \%$ were found to involve one muscle with an average diameter of $5.5 \mathrm{~cm} .^{2}$ Watson $e t a l^{5}$ found that $16 \%$ of all haemangiomas had more than one site.

Clinically, intramuscular haemangiomas usually present with pain $(55 \%)^{3}$ and swelling, with symptoms usually lasting one to five years (range 0-70 years). ${ }^{2}$ Haemangiomas may have a purpuric discolouration overlying (from cutaneous extension) the lesion. Superficial dilated veins may also be seen with cutaneous extension. A mass is found in $98 \%$ of cases. ${ }^{8}$ The mass may be pulsatile or have a bruit. Contraction of the muscle may increase the size of the lesion. The mass is usually moveable transversely but not in the line of the fibres. A history of trauma is uncommon $(17 \%)$, with tenderness and functional impairment found in about $25 \% .^{2}$ Over $90 \%$ are misdiagnosed before surgery (this was noted in 1957; advances in investigative techniques may have altered this figure). ${ }^{6}$ Complications may include a mass 
(direct pressure) effect, cardiac failure from arteriovenous shunting, and a consumptive coagulopathy (KasabachMerritt syndrome).

Calcified phleboliths may be seen on radiographs in $25 \%$ of cases. ${ }^{3}$ In one study by Derchi et al reported by Greenspan et al, ${ }^{9}$ ultrasound was effective in seven out of seven cases in diagnosing the intramuscular mass, with Doppler ultrasound not being very useful as the flow is usually too slow. It is sometimes difficult to assess the extent of the lesion with ultrasound. Magnetic resonance imaging has superseded other investigations, especially before surgery as it is noninvasive, can delineate the extent of the lesion, and can differentiate haemangioma from an invasive malignant process. ${ }^{10}$ Angiography is helpful in delineating whether there may be a vascular feeder that can be embolised. ${ }^{11} \mathrm{~A}$ group of patients investigated with computed tomography revealed that nine out of 12 lesions were more extensive when explored at surgery. ${ }^{12}$

A review by McNeill and Ray ${ }^{4}$ in 1974 of intramuscular haemangioma revealed:

- better outcome if lesion localised

- good results with complete excision

- partial excision resulted in continued symptoms

- limited success if treated non-operatively

- amputation is a last resort

Partial excision is associated with an $18 \%$ recurrence rate $^{13}$ because of the infiltrative growth pattern. None metastasised. ${ }^{13}$

Some have stated that radiation treatment may be associated with malignant transformation.

Compression sclerotherapy is another option in the treatment of intramuscular haemangioma. Agents that have been used include STS, ethanol, polidocanol, hypertonic saline, and sodium morrhuate. Studies comparing STS with ethanol and polidocanol in gastric varices indicate a similar efficacy. ${ }^{14}{ }^{15}$ Sclerotherapy is aimed at obliterating the lumen, with replacement of the patent vessel by fibrosis. Success of the treatment depends on many variables including extent of mural damage, thickness of vessel wall, amount of blood in the vein, duration of compression after treatment, sclerosing agent, and technique used In a review of 40 patients with craniofacial venous malformations, sclerotherapy resulted in clear improvement or cure in $75 \%$ and no or slight change in $25 \%{ }^{16}$ Winter et $a l^{17}$ treated 157 patients with haemangioma or vascular malformation with sclerotherapy (one to three injections in $86 \%$ of cases); $100 \%$ subjective satisfaction was reported. Potential complications include skin necrosis (blistering or ulceration), allergic reaction, bruising, hyperpigmentation, thrombosis of normal veins, neurolysis, and intra-arterial injection. Most complications are the result of extravasation of the sclerosant. ${ }^{18}$ Most complications are minor with a rate of less than $10 \% .{ }^{19}$ It is possible for the vessels to re-form channels.

Foamed sclerosant (formed by mixing liquid sclerosant 1:4 with air or carbon dioxide) is thought to produce better results than liquid sclerosant. Foamed sclerosant displaces blood from the vein, allowing a larger contact area, higher concentration, and longer contact time with the endothelium, which causes more intimal damage. With a more efficacious solution, the sclerosant concentration and therefore complications can be reduced. Foamed sclerosant has another advantage of being detectable by ultrasound, allowing visualisation of sclerosant placement closer to venous junctions. Using ultrasound guidance, the clinician can assess the extent of sclerosis (treatment success) as well as decreasing the risk of perivascular or unintentional intraarterial injection.
Take home message

- Differential diagnosis of pain and swelling in a muscle includes intramuscular haemangioma

- Ultrasound can diagnose the lesion but may not be able to delineate its extent

- The treatment of choice is total excision

- Sclerotherapy has a role in the management of intramuscular haemangioma when excision is not possible

Sclerotherapy may be used when:

- excision is not possible-for example, lesion is too large or where excision will destroy important tissue

- patient does not want surgery

- there is a need to debulk the tumour before surgery or cosmesis

STS exerts its effects by destroying the endothelium and exposing the highly thrombogenic factor VIII rich subendothelium to the circulation. ${ }^{20}$ It may also produce a hypercoagulable state by decreasing protein $\mathrm{C}$ and inducing platelets to aggregate. ${ }^{20}$ STS generally requires one to three injections $^{21}$ usually more than two weeks apart.22 Unlike ethanol, STS injection causes only minor pain and does not need a general anaesthetic. ${ }^{16}$

\section{OUTCOME}

In our patient, compression sclerotherapy (with STS under ultrasound guidance) was performed to both the symptomatic suprapatellar haemangioma and the lateral thigh haemangioma. Four injections were necessary. Currently he can participate in heavy training and competition without pain. The suprapatellar swelling and lateral thigh discolouration persists. The sclerosant therapy revealed that the two lesions actually communicated. There is an option for repeated injections.

\section{Authors' affiliations \\ R A Brown, K Crichton, Sydney Sports Medicine Centre, Sydney, Australia \\ G M Malouf, 236 Edgecliff Rd, Woolahra, NSW, Australia}

Correspondence to: Dr Brown, South Sydney Sports Medicine Centre, 111 Anzac Parade, Kensington, NSW 2033, Australia; rabrown4@ lycos.com

Accepted 28 April 2003

\section{REFERENCES}

1 Moser RP, Barr MS. Musculoskeletal case of the day. Intramuscular hemangioma of the thigh. AJR Am J Roentgenol 1994;162:1465-6.

2 Jenkins HP, Delaney PA. Benign angiomatous tumors of skeletal muscles. Surg Gynecol Obstet 1932;55:464-80.

3 Wild A, Raab P, Krauspe R. Hemangioma of skeletal muscle. Arch Orthop Trauma Surg 2000;120:139-43.

4 McNeill TW, Ray RD. Hemangioma of the extremities. Clin Orthop 1974; 101:154-66.

5 Watson WL, McCarthy WD. Blood and lymph vessel tumors: a report of 1056 cases. Surg Gynecol Obstet 1940;71:569.

6 Scott JES. Haemangiomata in skeletal muscle. Br J Surg 1957;44:496-501.

7 Tang P, Hornicek F, Gebhardt $M$, et al. Surgical treatment of hemangiomas of soft tissue. Clin Orthop 2002;1:205-10.

8 Ferguson IL. Hemangiomata of skeletal muscle. Br J Surg 1972;59:634.

9 Greenspan A, McGahan J, Vogelsang P, et al. Imaging strategies in the evaluation of soft-tissue hemangiomas of the extremeties. Skeletal Radiol 1992;21:11-18.

10 Weisberg AL, Haller JO, Wood BP. Radiological case of the month. Hemangioma of the thigh. Am J Dis Child 1989;143:379-80. 
11 Murakami M, Nonaka N, Hirata Y, et al. Hemangioma of the temporalis muscle: case report and review of the literature. Surg Neurol 1991;36:388-93.

12 Christenson JT, Gunterberg B. Intramuscular haemangioma of the extremities: is computerized tomography useful? Br J Surg 1985:72:748-50.

13 Allen PW, Enzinger FM. Hemangioma of skeletal muscle: an analysis of 89 cases. Cancer 1972;29:8.

14 Chawla Y, Dilawari J. Studies in sclerotherapy. 1. Comparison of sodium tetradecyl sulphate with absolute alcohol as sclerosants in the treatment of esophageal varices. J Clin Gastroenterol 1990;12:378-80.

15 Bhargava D, Singh B, Dogra R, et al. Prospective randomized comparison of sodium tetradecyl sulfate and polidocanol as variceal sclerosing agents. Am J Gastroenterol 1992;87:182-6.

16 Berenguer B, Burrows P, Zurakowski D, et al. Sclerotherapy of craniofacial venous malformations: complications and results. Plast Reconstr Surg 1999; 104:1-11.

17 Winter H, Drager E, Sterry W. Sclerotherapy for treatment of hemangiomas Dermatol Surg 2000;26:105-8.
18 Green D. Compression sclerotherapy techniques. Dermatol Clin 1989;7:137-46.

19 Frullini A, Cavezzi A. Sclerosing foam in the treatment of varicose veins and telangiectases. History and analysis of safety and complications. Dermatol Surg 2002;28:11-15.

20 Jacobson B, Franz R, Hurly E, et al. Mechanism of thrombosis caused by sclerotherapy of esophageal varices using sodium tetradecyl sulphate. Surg Endosc 1992;6:4-9.

21 Siniluoto T, Svendsen P, Wikholm G, et al. Percutaneous sclerotherapy of venous malformations of the head and neck using sodium tetradecyl sulphate. Scand J Plast Reconstr Hand Surg 1997;31:145-50.

22 Anavi Y, Har-El G, Mintz S. The treatment of facial haemangioma by percutaneous injections of sodium tetradecyl sulfate. J Laryngol Otol 1988; 102:87-90.

23 Nack J, Gustafson L. Intramuscular hemangioma. J Am Podiatr Med Assoc 1990;8:441-3.

24 Rogalski R, Hensinger R, Loder R. Vascular abnormalities of the extremities: clinical findings and management. J Pediatr Orthop 1993;13:9-14. 